\title{
Self-Monitoring of Blood Glucose in Children and Adolescents with Diabetes- Barriers, Behaviors, and the Search for Solutions
}

\author{
a report by \\ William A Fisher, PhD $^{1}$ and Holly Schachner, MD ${ }^{2}$ \\ 1. Professor of Psychology, and Professor of Obstetrics and Gynecology, University of Western Ontario; \\ 2. Director of Medical and Clinical Affairs, Diabetes Care, Bayer Healthcare
}

DOI: 10.17925/USE.2008.04.2.71

Diabetes is a common and consequential chronic disease of childhood. Estimates of the prevalence of diabetes among children and adolescents under 20 years of age in the US range from $154,000^{1}$ to $200,000,{ }^{2}$ with type 1 diabetes (annual incidence of 15,000 ) predominating among younger children and type 2 diabetes (annual incidence 3,700) increasing among adolescents. Some $0.88 \%$ of males and $1.12 \%$ of females will develop diabetes by 20 years of age, ${ }^{3}$ with important racial and ethnic differences observed in the prevalence of type 1 and type 2 diabetes among children and youth. ${ }^{1}$ It has been estimated that among children diagnosed with diabetes at 10 years of age, boys will lose 18.7 life-years and 31.0 quality-adjusted life-years, while girls will lose 17.9 life-years and 31.8 quality-adjusted life-years. ${ }^{3}$

Guidelines for the care of children and adolescents with diabetes provide comprehensive information concerning the clinical and developmental needs of children at varying stages of maturity and development (see Silverstein et al. ${ }^{4}$ for guidelines on the care of children and adolescents with type 1 diabetes, and see the American Diabetes Association [ADA $]^{5}$ and Rosenbloom et al. ${ }^{6}$ for discussion of the emerging epidemic of type 2 diabetes and guidelines for its treatment in youth). On the basis of the Diabetes Control and Complications (DCCT) trial7,8 and follow-up studies $^{9,10}$ showing a significant relationship between glycemic control and subsequent development of complications of diabetes, recommendations call for intensive management of diabetes in children and youth with the goal of attaining as near normal glucose control as possible. With respect to diabetes management for pediatric and adolescent patients with type 1 diabetes, however, Silverstein et al. ${ }^{4}$ note that "extensive evidence indicates that near-normalization of blood glucose levels is seldom attainable in children and adolescents after the honeymoon (remission) period." With respect to adolescents with type 1 diabetes, Silverstein et al. ${ }^{4}$ emphasize that "enthusiasm for embracing the target achieved by the intensively treated adult cohort of the DCCT is tempered by the recent results of Epidemiology of Diabetes Interventions and Complications ${ }^{8} \ldots$ When the cohort of adolescents included in the DCCT was analyzed separately, ${ }^{8}$ the $\mathrm{A} 1 \mathrm{C}$ level achieved in the 'intensive' group was $>1 \%$ higher than the current ADA recommendation for patients in general. ${ }^{11 "}$ Moreover, follow-up studies of the DCCT cohort ${ }^{4,9}$ show that adolescents who were treated intensively within the DCCT trial showed reduced glycemic control when treated intensively outside of the trial context, while adolescents with type 1 diabetes treated with conventional therapy within the trial had increased glycemic control when treated intensively outside of the clinical trial setting. At the same time, however, both groups showed reductions in the worsening of complications of diabetes over time. ${ }^{4,9}$ Accordingly, investigators have concluded that the findings support intensive therapy for adolescents with type 1 diabetes with the goal of attaining near normal glycemic control in order to reduce the risk for microvascular and macrovascular complications. ${ }^{4,9}$

\section{Diabetes Management in Children and Adolescents-}

Clinician, Patient, and Parent Perspectives

From the clinician's perspective, when faced with sending home a child newly diagnosed with diabetes from the hospital or office, one of the easiest (yet tedious) tasks is writing out all of the patient's new prescriptions. Writing these prescriptions has become quite rote: lancetsthe smallest and finest for the smallest and most delicate hands; blood glucose monitor and strips to check blood glucose results before each meal, at bedtime, and at times overnight, as well as to monitor for suspected low readings and anticipated lows for a new sports season or other such change; and medication to treat the results of blood glucose monitoring-always insulin for children and adolescents with type 1 diabetes, and sometimes insulin for those with type 2 diabetes, as well as other medications that may or may not have been fully studied in children. If insulin is prescribed, both long-acting and short-acting will be needed, depending on blood glucose results and time of day, as well as needles to inject the insulin (again, perhaps the smallest and finest) and glucose tablets to treat low blood glucose levels. A glucagon emergency kit will also be needed for lows so severe that they may prevent a parent or child from ever attempting to reach glycemic control targets again.

In the weeks and months that follow initial diagnosis, there will be regular calls about insulin and medication adjustment, nutritional guidance, meal planning, and sick-day management (children and youth with diabetes are not immune to the flu or strep throat), as well as discussions about daily routines, special events, and changes and challenges due to glycemic

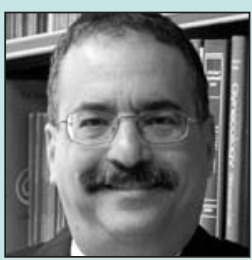

William A Fisher, PhD, is a Professor of Psychology and a Professor of Obstetrics and Gynecology at the University of Western Ontario, and a Research Affiliate at the Center for Health, Intervention, and Prevention at the University of Connecticut. He has held a National Health Scientist award from Health Canada, has served on the Editorial Boards of seven academic journals, and has published over 170 papers in the area of health behavior and health behavior change. His research has been supported by the US National Institutes of Health $(\mathrm{NIH})$ for the past two decades. Professor Fisher has been a lead investigator on a series of health behavior prediction and intervention studies based on Fisher and Fisher's 1992 Information-Motivation-Behavioral Skills model of health behavior change, as well as on the MALES and FEMALES research programs focusing on male and female sexual dysfunction and comorbid medical illness.

E: fisher@uwo.ca 
Figure 1: An Information-Motivation-Behavioral Skills Model of Self-monitoring of Blood Glucose

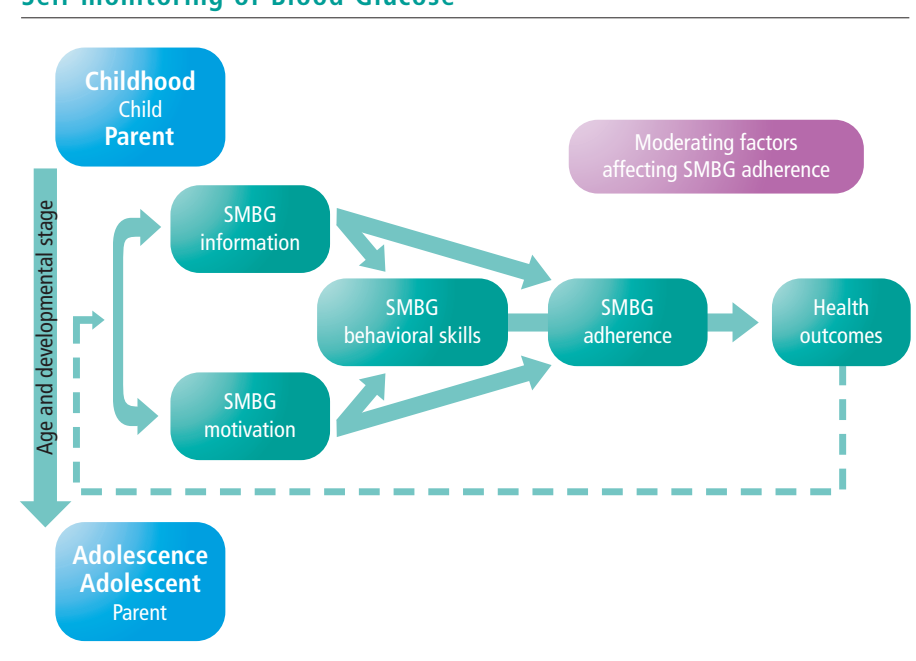

Source: Fisher et al., 2006, ${ }^{22}$ and Fisher et al., 2003.21

control associated with the child or adolescent's normal growth and development. There may also be further discussions about insulin pump therapy (see Ahern et al. $^{12}$ and Plotnick et al..$^{13}$ ) and continuous glucose monitoring (see Chase et al. ${ }^{14}$ and Boland and Tamborlane ${ }^{15}$ ) and how to use these technologies to help assess and manage daily metabolic control needs. What may be the clinician's most difficult task, however, is helping patients, parents, and families incorporate the demands of diabetes management into their daily lives without disrupting the hopes and dreams that children and adolescents with diabetes and their families had prior to the diagnosis of diabetes.

From the perspective of children and adolescents and their parents, the diagnosis of diabetes presents a number of immediate, challenging, and ongoing demands. First, it is necessary for the young patient and his or her parents to acquire a complicated and novel body of information that must be acquired, understood, and translated into the daily actions required to effectively implement diabetes care. Second, it will be essential for children, adolescents, and parents to maintain a critical level of personal motivation sufficient to support intensive diabetes management over the long run and to recruit social support from family, other care-givers, and friends for carrying out the many tasks involved in care for this chronic condition. In addition, it will be essential for children and adolescents with diabetes and their parents to develop a set of behavioral skills to cope effectively with the complex health behavior demands of intensive management of diabetes over time, and to deal with factors in the patient, family, and surrounding environment that may work against management of diabetes.

As noted earlier, a cornerstone of management of diabetes in children and adolescents is maintenance of glycemic control at as close to normal levels as possible. ${ }^{4,5,9}$ At present, the basis for glycemic control involves adherence to self-monitoring of blood glucose (SMBG) and translation of SMBG readings into effective management steps to maintain glycemic control (see Saudek et al., ${ }^{16}$ Fisher et al., ${ }^{17}$ Johnson et al., ${ }^{18}$ and Mancuso et al. ${ }^{19}$ see also Boland et al. ${ }^{20}$ for limitations of SMBG). As with all other aspects of intensive management of diabetes over the long term, children and adolescents with diabetes and their parents will need relevant information about SMBG, motivation to adhere to the SMBG regimen, and behavioral skills for adhering effectively to SMBG recommendations. The diabetes management demands for SMBG faced by children and adolescents with diabetes and their parents are represented within the Information-Motivation-Behavioral Skills (IMB) model of health behavior ${ }^{21,22}$ (see Figure 1), which can be applied to help understand and promote diabetes care among children and adolescents and in the family context.

\section{Understanding Self-monitoring of Blood Glucose Adherence-An Information-Motivation-Behavioral Skills Analysis}

According to the IMB model, SMBG information is a prerequisite for adherence to recommended SMBG frequency, to understanding SMBG results, and to undertaking appropriate actions based on SMBG readings. Patients and parents (whose capabilities and responsibilities vary as a function of the child or adolescent's age and developmental stage) must master a considerable body of SMBG information, including how to test (which will include meter and strip use, calibration, recording of results, and myriad other items of information), when to test, and what health intervention actions to take in relation to high or low SMBG readings. ${ }^{19}$ In addition to these discrete items of information, we note that SMBG heuristics, or simple and cognitively effortless decision rules, may also influence SMBG adherence, for better or for worse. For example, if a young patient or parent concludes that an acceptable glycated hemoglobin $\left(\mathrm{HbA}_{1 \mathrm{c}}\right)$ result indicates adequate SMBG adherence, suboptimal SMBG adherence may result. Alternately, in young patients or parents who automatically undertake SMBG at the recommended times because it is time to do so, more optimal SMBG adherence is likely to result.

From the perspective of the IMB model, SMBG motivation is an additional prerequisite that will determine whether even well-informed young patients and parents will act on what they know to adhere to SMBG recommendations. Personal motivation to adhere to SMBG may be based on beliefs about the outcomes of adherence (e.g. 'it will help me or my child avoid complications of diabetes,' 'it will be painful and disruptive') and the individual's positive to negative evaluation of these outcomes ${ }^{21,23}$ (see DeLamater et al. ${ }^{24}$ and Skinner and Hampson ${ }^{25}$ for discussion of the relationship of beliefs about the seriousness of diabetes, vulnerability to complications, costs of adherence to regimen, and beliefs about treatment efficacy with regimen adherence and glycemic control). Social motivation to adhere to SMBG may rest on perceptions of the wishes of significant others (e.g. parents, physicians, diabetes educators) and the individual's motivation to comply with or to defy the wishes of significant others (see DeLamater et al. ${ }^{24}$ and DeLamater ${ }^{26}$ for a discussion of social influence of the family on regimen adherence and glycemic control, and see Kyngas et al. ${ }^{27}$ for research concerning the influence of perceived social support from physicians, nurses, parents, and friends and adherence to diabetes self-care).

Following an IMB model analysis, SMBG behavioral skills comprise an additional critical factor that will determine whether even well-informed and well-motivated patients and parents can adhere effectively to SMBG recommendations. SMBG behavioral skills may involve objective abilities as well as a subjective sense of capability for performing a complex 
sequence of SMBG-related behaviors (see DeLamater et al. ${ }^{24}$ for a discussion of the relationship between self-efficacy, learned helplessness, and glycemic control, see Kaufman et al. ${ }^{28}$ for research concerning the relationship of family behavioral skills competencies and metabolic control among children with diabetes, and see Lemanek et al. ${ }^{29}$ for evidence of effectiveness of interventions that include behavioral skills training content). The SMBG behavioral sequence may involve steps such as keeping an SMBG meter accessible for use when needed, self-cueing SMBG before meals and at bedtime, and skills for relatively comfortably drawing a blood drop, using a meter to test blood glucose levels accurately, taking appropriate action contingent on blood glucose reading, recording blood glucose levels, discerning patterns in blood glucose readings, and bringing up and discussing blood glucose readings with the diabetes care clinicians. A parallel set of behavioral skills for prompting and guiding children's and adolescent's diabetes management effectively while minimizing family conflict may also be essential for adherence to SMBG among children and adolescents with diabetes.

According to the IMB model (see Figure 1), SMBG information and SMBG motivation work through the application of SMBG behavioral skills to effect SMBG adherence. Well-informed and well-motivated child and adolescent patients and their parents will have to apply SMBG behavioral skills in order to adhere to SMBG recommendations. In cases where SMBG behavioral skills are not necessary to effect SMBG adherence, direct links between SMBG information and SMBG motivation with SMBG behavior may be seen, but under the current circumstances SMBG behavioral skills on the part of young patients and parents would appear to be essential to SMBG adherence.

A number of other critical factors are addressed in the IMB model of SMBG adherence. As shown in Figure 1, there is a feedback loop such that SMBG adherence will influence health outcomes such as subjective feelings of good or ill health, $\mathrm{HbA}_{1 \mathrm{c}}$ levels, and complications of diabetes. In theory, positive health outcomes and self, parent, and clinician reinforcement of positive outcomes will strengthen the application of SMBG information, SMBG motivation, and SMBG behavioral skills and the maintenance of SMBG adherence over time. The feedback loop can, however, be misleading in cases where an individual who is not SMBGadherent nonetheless experiences subjective feelings of health and concludes that current levels of SMBG are sufficient. SMBG health outcomes that involve preoccupation with physical discomfort associated with testing or preoccupation with one's chronic illness occasioned by testing may also have a negative influence on SMBG adherence over time.

An additional factor meriting close attention in the IMB analysis of SMBG adherence involves moderating factors in the environment that may directly influence adherence to monitoring. Environmental factors as diverse-and important-as day-care arrangements, school attendance, family structure, family conflict, and insurance coverage may all influence SMBG adherence, and research has specifically and repeatedly demonstrated a link between family conflict, diabetes regimen adherence, and metabolic control (see DeLamater et al., ${ }^{24}$ Anderson et al., ${ }^{30}$ MillerJohnson et al., ${ }^{31}$ Pattonet al., ${ }^{32}$ and DeLamater ${ }^{26}$ for discussion of environmental and family factors and metabolic control). Moreover, as indicated in Figure 1, age and developmental stage are expected to significantly influence capabilities and responsibilities for SMBG adherence (see Silverstein et al. $^{4}$ for a thorough discussion of the role of age and development in relation to diabetes self-care and the roles of children and adolescents with diabetes and their parents). As noted in Figure 1, during childhood parents' SMBG information, motivation, and behavioral skills will be of paramount importance in ensuring the child's SMBG adherence and appropriate actions taken on the basis of blood glucose readings. The parental role will transition to a somewhat less prominent position as the child enters adolescence and his or her SMBG information, motivation, and behavioral skills play an increasingly significant role in SMBG adherence and glycemic control, with maintenance of appropriate parental input and supervision and a hoped-for minimization of conflict with the adolescent. ${ }^{4,26,30}$ Research has indicated that children may have fewer problem-solving skills than adolescents, while paradoxically having better regimen adherence, potentially due to developmental differences in factors as diverse as degree of parental supervision of childhood diabetes and degree of adolescent vulnerability to social pressure situations (see Thomas et al., ${ }^{33}$ Silverstein et al., ${ }^{4}$ and Anderson et al. ${ }^{34}$ for discussion of these issues). Halvorson et al..$^{35}$ provide an extensive discussion of developmental considerations and age-specific management challenges for children and adolescents with diabetes.

According to the Information-

Motivation-Behavioral Skills (IMB)

model, self-monitoring of blood

glucose (SMBG) information and

SMBG motivation work through the

application of SMBG behavioral skills to

effect SMBG adherence.

Promoting Self-monitoring of Blood Glucose Adherence and Achieving Metabolic Control-The Search for Solutions

The IMB model analysis of determinants of SMBG adherence suggests that efforts to provide relevant information, strengthen personal and social motivation, and teach and refine behavioral skills for SMBG adherence and translation of blood glucose readings into appropriate action will be effective in assisting children, adolescents, and parents in achieving metabolic control. Such information, motivation, and behavioral skills interventions should represent a co-ordinated effort by a diabetes care team with medical, educational, psychological, and behavioral expertise; they must be appropriate to the developmental stage and evolving capacities of the children and adolescents involved, and they must address environmental challenges, including family conflict, to adherence to SMBG and metabolic control.

A considerable number of interventions to support SMBG adherence and glycemic control among children and adolescents with diabetes have been reported in the literature (see meta-analytic and narrative reviews by Hampson et al., ${ }^{36}$ Lemanek et al., ${ }^{29}$ Grey et al., ${ }^{37}$ Winkely et al., ${ }^{38}$ and DeLamater ${ }^{26}$ ). Results of adherence support and glycemic control interventions have generally been consistent with the assumptions of the IMB model concerning the critical roles of information, motivation, and behavioral skills in SMBG adherence. In a meta-analysis of behavioral 
interventions for adolescents with type 1 diabetes, ${ }^{36}$ skills-training efforts, family-related interventions, dietary interventions, and problem-solving interventions, in descending order, were the most common intervention efforts reported. Meta-analysis of intervention impact found a moderate effect size for theory-based interventions (mean effect size 0.47 ) and a much smaller mean effect size for atheoretical interventions (mean effect size 0.06). Importantly, overall intervention effects on psychosocial outcomes (e.g. self-efficacy, communication skills; mean effect size 0.37) and blood glucose levels (mean effect size 0.33 ) were positive and similar.

An additional recent meta-analysis of the research literature concerning interventions for children and adolescents with type 1 diabetes, ${ }^{38}$ most involving elements of cognitive behavioral therapy, reported a significant mean effect size of 0.35 on outcomes relevant to diabetes management. Despite the uneven methodological quality of the studies reviewed, a mean percentage reduction of $\mathrm{HbA}_{1 \mathrm{c}}$ of $0.48 \%$ - sufficient to reduce progression to microvascular complications-was reported. Similarly, a narrative review of 11 interventions to promote adherence to a diabetes management regimen in children and adolescents ${ }^{29}$ reported that multicomponent and direct reinforcement of regimen-adherence interventions met criteria of probable efficaciousness, and cognitive behavioral interventions appeared promising according to published efficacy criteria.

Individual studies reporting a significant impact on regimen adherence and metabolic outcome include a randomized trial by Ellis et al. ${ }^{39}$ in which multisystemic therapy ("an intensive, family-centered, community-based treatment") with adolescents with type 1 diabetes and chronically poor metabolic control and their families was effective in increasing the frequency of SMBG and decreasing inpatient hospital admissions, and provided evidence of increased metabolic control (see also Wysocki et al. ${ }^{40}$ for a report of the impact of family therapy on adherence to regimen). Similarly, Svoren et al. ${ }^{41}$ conducted a randomized two-year prospective trial in which 'Care Ambassadors' (case managers who assisted with appointment scheduling and insurance issues), with or without psychoeducational intervention modules including information, motivation, and behavioral skills content, or standard of care, were compared with respect to diabetes management outcomes. The Care Ambassador plus psychoeducational intervention resulted in significantly reduced rates of short-term adverse outcomes, $60 \%$ fewer severe hypoglycemic events, and 40\% fewer hospitalizations and emergency department visits compared with the other conditions. Moreover, adolescents with baseline $\mathrm{HbA}_{1 \mathrm{c}}$ levels $>8.6 \%$ in the Care Ambassador plus psychoeducational intervention condition had a 3.4-fold greater likelihood of improved glycemic control than those with similar baseline $\mathrm{HbA}_{1 \mathrm{c}}$ in other conditions.

An office-based intervention to improve parent-adolescent teamwork ${ }^{42}$ and an intervention targeting improvement of blood glucose management in the school setting for children with diabetes ${ }^{43}$ have also been found to improve metabolic control. Other interventions, such as a telephone-based intervention to provide support and development of problem-solving skills among adolescents with type 1 diabetes ${ }^{44}$ and a peer group intervention with adolescents with type 1 diabetes and their best friends, ${ }^{45}$ showed improvements in self-efficacy and in peer support, respectively, although no changes in metabolic control were reported. Evidence that intervention effects may not automatically persist beyond enrollment in a clinical trial ${ }^{7,8,46}$ strongly suggests that clinical and research attention to maintenance of intervention impact is needed. Overall, however, a recent overview of the intervention literature provided the optimistic conclusion that the results of intervention studies "indicate that family-based behavioral procedures such as goal-setting, self-monitoring, positive reinforcement, behavioral contracts, supportive parental communications, and appropriately shared responsibility for diabetes management have improved regimen adherence and glycemic control." 26

\section{Conclusion}

The current discussion suggests that the effective management of diabetes in children and adolescents occurs at the junction of human biology and human behavior. While research-based advances in intensive management of diabetes continue, with SMBG adherence and glycemic control representing cornerstones of care, young patients, their parents, and their clinicians must possess information, motivation, and behavioral skills for implementing and supporting complex diabetes management actions over time and in the context of developmental, family, and environmental factors that may be supportive or disruptive. The diabetes clinical care team requires expertise in ongoing educational and supportive efforts across the childhood and adolescent development spectrum to assist with the medical and behavioral challenges of intensive diabetes management in this age range.
1. SEARCH for diabetes in Youth Study, Pediatrics, 2006;118:1510-18.

2. www.cdc.gov/DIABETES/pubs/estimates.htm\#prev2

3. Narayan KMV, et al., JAMA, 2003;290(14):1884-90.

4. Silverstein J, et al., Diabetes Care, 2005;28(1):186-212.

5. American Diabetes Association, Diabetes Care, 2000;23(3):381-9.

6. Rosenbloom AL, et al., Diabetes Care, 1999;22:345-54.

7. The Diabetes Control and Complications Trial Research Group, N Engl J Med, 1993;329(13):977-86.

8. Diabetes Control and Complications Trial Research Group, J Pediatr, 1994;125(2):177-88.

9. Diabetes Control and Complications Trial (DCCTP)/Epidemiology of Diabetes Interventions and Complications (EDIC) Research Group, J Pediatr, 2001;139(6):804-12.

10. The Epidemiology of Diabetes Interventions and Complications (EDIC) Study, JAMA, 2003;290(14):2159-67.

11. American Diabetes Association, Diabetes Care, 2004;27(Suppl. 1): S15-S35.

12. Ahern JH, et al., Pediatr Diabetes, 2003;3:10-15.

13. Plotnick LP, et al., Diabetes Care, 2003;26(4):1142-6.
14. Chase HP, et al., Pediatrics, 2001;107(2):222-6.

15. Boland EA, Tamborlane WV, Diabetes Technol Ther, 2000;2(1):53-9.

16. Saudek CD, et al., JAMA, 2006;295(14):1688-97.

17. Fisher WA, US Endocrine Disease, 2007;51-3.

18. Johnson SB, et al., J Pediatr Psychol, 1992;17(5):537-53.

19. Mancuso M, et al., Acta Biomed, 2005;3:56-8.

20. Boland E, et al., Diabetes Care, 2001;24(11):1858-62.

21. Fisher WA, et al.. In: Suls J, Wallston K (eds), Social psychological foundations of health and IIIness, London: Blackwell, 2003;82-106.

22. Fisher JD, et al., Health Psychol, 2006;25:462-73.

23. Fishbein M, Ajzen I, Belief, attitude, intention, and behavior. An introduction to theory and research, Reading, MA: Addison-Wesley, 1975.

24. Delamater AM, et al., Diabetes Care, 2001;24(7):1286-92.

25. Skinner TC, Hampson SE, Diabetes Care, 2001;24(5):828-33.

26. DeLamater AM, Pediatr Diabetes, 2007;8:340-48.

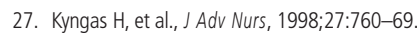

28. Kaufman FR, et al., Pediatr Diabetes, 2002;3:179-83.

29. Lemanek KL, et al., J Pediatr Psychol, 2001;6(5):253-75.
30. Anderson BJ, et al., Diabet Med, 2002;19:635-42.

31. Miller-Johnson S, et al., J Consult Clin Psychol, 1994;62(3): 603-10.

32. Patton SR, et al., Diabetes Care, 2006;29(5):1002-6.

33. Thomas AM, et al., J Pediatr Psychol, 1997;22(4):541-61.

34. Anderson B, et al., J Pediatr, 1997;130(2):257-65.

35. Halvorson M, et al., Diabetes Spectrum, 2005;18(3):167-73.

36. Hampson SE, et al., Diabetes Care, 2000;23(9):1416-22.

37. Gray M, et al., Diabetes Education in Children, 1999;25(6): 25-33.

38. Winkley K, et al., Research, 2006;1-5.

39. Ellis DA, et al., Diabetes Care, 2005;28(7):1604-10.

40. Wysocki T, et al., Diabetes Care, 2001;24(3):441-6.

41. Svoren BM, et al., Pediatrics, 2003;112(4):914-22.

42. Anderson BJ, et al., Diabetes Care, 1999;22(5):713-21.

43. Nguyen TM, et al., J Pediatr, 2008;153(4):575-8.

44. Howells L, et al., Diabetic Medicine, 2002;19:643-8.

45. Greco P, et al., J Pediatr Psychol, 2001;26(8):485-90.

46. Couper JJ, et al., Diabetes Care, 1999;22(12):1933--7. 